\section{EL PROCESO \\ VOCACIONAL DE PEDRO}

Autor: Santiago Guijarro Oporto es Sacerdote Operario Diocesano. Profesor de Nuevo Testamento en la Universidad Pontificia de Salamanca y Director Espiritual del Seminario Interdiocesano

"Santiago Apóstol"

\section{DOI: https://doi.org/10.52039/seminarios.v47i160.968}

La experiencia vocacional ocupa un lugar destacado en los libros de la Biblia, y por esta razón se recurre a ella con frecuencia para identificar y discernir la llamada de Dios. De todos los pasajes de la Biblia, los "relatos de vocación" son, probablemente, los más conocidos y también los más utilizados en el proceso de discernimiento vocacional, porque en ellos aparecen con claridad y de forma insistente los rasgos propios de la llamada de Dios. Ahora bien, en la mayoría de estos relatos la vocación aparece como un hecho puntual, mientras que en nuestra experiencia acontece como un proceso, como una sucesión de llamadas, que tienen matices distintos en las distintas edades de la vida. Se trata de una contradicción más aparente que real, pues también los relatos bíblicos presuponen que la vocación es un proceso. La experiencia vocacional de Pedro, tal como aparece en los evangelios, es un buen ejempío de elío, como espero mostrar en las páginas que siguen.
La experiencia vocacional de Pedro es un buen ejemplo de que la vocación es un proceso, condensado en el relato. Los momentos fuertes se relacionan con las edades de la vida. Estudia el autor dos textos. La primera llamada a orillas del lago: «os haré pescadores de hombres» y la hecha a través de Andrés (In 1,35.42). En el segundo momento (Me 8) después de haberlo confesado como Mesías, Jesús le indica que aún no está maduro, sería ultimo episodio (In 21) destaca la primacía del amor en el diálogo vocacional. 


\section{La experiencia vocacional como proceso}

Cuando leemos los relatos de vocación tenemos la impresión de que Moisés, Isaías o Jeremías experimentaron la llamada de Dios en un

El relato vocacional condensa un largo proceso

El relato es la sintesis de un proceso. Escrito al final del mismo momento concreto de su vida (al acercarse a la zarza ardiendo, mientras estaba en el templo...) y de una vez para siempre. Nos llama la atención la rapidez con que responden a dicha llamada, a pesar de las dificultades e inconvenientes que descubren. En un momento, generalmente muy breve, se ha dado un proceso que a la mayoría de nosotros nos ha costado años. Pero, ¿fue acaso su experiencia tan distinta de la nuestra?

Probablemente no. Es la impresión que recibimos cuando olvidamos que los relatos de vocación no son una crónica de la experiencia vocacional, sino un género literario, que condensa una experiencia más amplia. Hace ya casi treinta años G. del Olmo estudió con detalle estos relatos y demostró que han sido formulados con ayuda de moldes literarios muy parecidos. Una de las consecuencias de su estudio es que hay que distinguir entre la experiencia vocacional y su formulación en los relatos de vocación ${ }^{1}$. Dicha formulación tuvo lugar casi siempre al final de la vida del protagonista, e incluso después de su muerte, de modo que en ella se encuentra recogida no sólo la llamada inicial, sino una experiencia más dilatada y compleja: todo su proceso vocacional.

Si leemos los relatos de vocación como la síntesis de un proceso, muchos de sus aspectos llamativos nos resultarán más fácilmente comprensibles. Hubo una llamada puntual al principio, pero esta llamada se fue explicitando con el tiempo. Hubo también una respuesta inicial, pero sólo con el paso del tiempo llegó a ser tan firme y decidida como reflejan los relatos de vocación.

Podemos recurrir a dos ejemplos para ilustrar esta relación entre la experiencia vocacional y su formulación posterior: el de Jeremías y el de Pablo. En el libro de Jeremías encontramos dos versiones de su vocación: la del relato inicial, construido según las pautas de los relatos de vocación (Jer 1,4-10), y la de sus "confesiones" repartidas a lo

${ }^{1} \mathrm{G}$. del Olmo, La vocación del líder en el antiguo Israel. Morfología de los relatos bíblicos de vocación (Valencia: Institución San Jerónimo 1973). El estudio se ocupa casi exclusivamente de la forma de los relatos de vocación, pero el autor reconoce que detrás de ellos hay una experiencia vivida, probablemente más compleja y variada. Véanse pp. 416419. 
largo del libro (Jer 11,18-12,6; 15,10-21, 17,14-18; 18,18-23; 20,7-18). Esta segunda versión está más cerca de cómo vivió Jeremías la llamada de Dios, y en ella aparece claramente que la experiencia vocacional es un proceso, en el que la respuesta a Dios debe renovarse constantemente. Lo mismo podemos observar en las dos versiones que tenemos de la experiencia vocacional de Pablo: la suya propia, recogida básicamente en Gál 1,11-2,10; y la de sus discípulos, contada tres veces en el libro de los Hechos (Hch 9,1-30; 22,6-16; 26,12-18). Al compararlas observamos que el testimonio de Pablo describe un largo proceso, mientras que el relato de sus discípulos narra un hecho puntual.

La vocación es, pues, un proceso con algunos momentos fuertes que jalonan el camino de una relación continuada entre Dios y quienes reciben su llamada. La psicología religiosa nos revela que los momentos fuertes de la experiencia vocacional suelen coincidir con las diversas fases de la experiencia espiritual, que a su vez poseen una estrecha relación con las edades de la vida ${ }^{2}$. Esto es, precisamente lo que encontramos en las escenas de los evangelios que recogen diversos momentos de la experiencia vocacional de Pedro.

\section{La experiencia vocacional de Pedro según los evangelios}

Pedro es, después de Jesús, el personaje del que más se habla en los evangelios. Su lugar en el grupo de los Doce, y la relevancia que tuvo más tarde en las comunidades cristianas explican este protagonis$\mathrm{mo}^{3}$. Aquí vamos a considerar un aspecto al que no suele prestarse mucha atención: su experiencia vocacional, que es singular no sólo en el conjunto del Nuevo Testamento, sino de toda la Biblia4.

${ }^{2}$ Una presentación de estas relaciones, sobre las que volveremos más adelante con más detalle, puede verse en: J. Garrido, Adulto y cristiano. Crisis de realismo y madurez cristiana (Santander: Sal Terrae 1989, $2^{\text {a }}$ ed.) pp. 73-84.

3 Ambas cosas han sido el principal objeto de los estudios petrinos, sobre los que existen dos publicaciones conjuntas y bastante representativas en castellano: R. E. Brown K. P. Donfried - J. Reuman, Pedro en el Nuevo Testamento. Palabra Inspirada 15 (Santander: Sal Terrae 1976); y: R. Aguirre (ed.), Pedro en la iglesia primitiva (Estella: Verbo Divino 1991).

${ }^{4}$ El único estudio que conozco dedicado específicamente a los relatos de la vocación de Pedro es el de S. O. Abogunrin, "The Three Accounts of Peter's Call: a Critical and Theological Examination of the Texts" New Testament Studies 31 (1985) 587-602. 
Cuando se estudia la vocación de Pedro suelen mencionarse dos pasajes. El más conocido es, sin duda, el de Marcos: Jesús va caminando por la orilla del lago y se encuentra a Pedro y a su hermano

Encuentro a la orilla del lago. Encuentro cerca del Jordán Andrés que están echando las redes, les invita a seguirle con un dicho enigmático (convertirse en pescadores de hombres), y ellos, dejando las redes, le siguen (Mc 1,16-18)5. El otro relato se encuentra en el evangelio de Juan, y difiere notablemente del anterior. En este caso no es Jesús quien atrae la atención de Pedro, sino su hermano Andrés, que acaba de conocer a Jesús gracias al testimonio del Bautista (Jn 1,3542). Las notables diferencias que existen entre estos dos relatos han planteado numerosas preguntas sobre la vocación inicial de Pedro: ¿Tuvo lugar en Galilea o en Judea? ¿Fue Andrés quien llevó a Pedro hasta Jesús, o Jesús se encontró con ambos al mismo tiempo? ¿Qué fue lo que Jesús le dijo a Pedro? La mayoría de estas preguntas presuponen que los dos pasajes se refieren al mismo acontecimiento, pero es probable que Juan y Marcos hayan conservado dos tradiciones distintas acerca del primer encuentro de Pedro con Jesús.

Además de estos dos pasajes hay otros dos en los que explícitamente se habla de la experiencia vocacional de Pedro, aunque casi nunca se estudian desde esta perspectiva. El primero de ellos se encuentra en un lugar estratégico del evangelio de Marcos. Al acercarse a Cesarea de Filipo, Jesús pregunta a sus discípulos qué dice la gente acerca de él, y qué es lo que piensan ellos. Pedro le responde reconociéndole como Mesías. Jesús trata de corregir esta afirmación anunciando por primera vez su inminente pasión, pero Pedro le increpa en señal de desaprobación. Jesús, entonces, le invita a que vuelva a ocupar su lugar como discípulo (Mc 8,27-33). Estas últimas palabras de Jesús evocan de algún modo la primera llamada, pues en ambos casos se utiliza la expresión "detrás de mî́, que es un término técnico del discipulado en los evangelios. Este episodio refleja, por tanto, una

${ }^{5}$ Este pasaje tiene una paralelo claro en Mateo (Mt 4,18,20), y otro menos evidente en Lucas (Lc 5,10-11), pero ambos dependen de Marcos. Abogunrin, "The Three Accounts... pp. 590-594 piensa que Lc 5,1-11 representa una tradición independiente de la llamada inicial de Pedro. Sin embargo, Lc 5,10-11 puede explicarse fácilmente como una reelaboración lucana de Mc 1,16-18. Véase: S. Guijarro Oporto, Fidelidades en conflicto. La ruptura con la familia por causa del discipulado y de la misión en la tradición sinóptica. Plenitudo Temporis 5 (Salamanca: Universidad Pontificia 1998) pp. 174-178. 
nueva invitación de Jesús a Pedro, en un momento clave de su itinerario como discípulo6.

El otro pasaje se encuentra al final del evangelio de Juan, en un capítulo que fue añadido tardíamente al evangelio. La escena que nos interesa tiene lugar en el marco de un largo encuentro de Jesús con Pedro. Después de la escena de la pesca milagrosa (Jn 21,1-14), Jesús se queda sólo con Pedro y por tres veces le pregunta sobre su actitud hacia él. Cuando Pedro le ha confesado reiteradamente su amor, Jesús le anuncia veladamente su muerte y le invita a seguirle (Jn 21,15-19). El contexto es claramente vocacional, porque el verbo "seguir" que se utiliza aquí es el otro verbo técnico del discipulado. En otros lugares del evangelio de Juan la relación de Pedro con Jesús se describe a través de este mismo verbo (Jn 13,36-37, y de forma más velada en Jn 18,15), pero sólo en este lugar encontramos en boca de Jesús una invitación formulada en imperativo: “¡Tú, sígueme!”.

Así pues, tenemos en total cuatro episodios que pueden ser ambientados en diversos momentos del ministerio de Jesús, y por tanto de la relación que Pedro tuvo con él.

En Judea, antes del arresto de Juan Bautista (Jn 1,41-42).

En Galilea, después de que Juan fuera arrestado (Mc 1,16-18).

En Cesarea de Filipo, después del ministerio en Galilea (Mc 8,33).

En Galilea, después de que Jesús resucitara (Jn 21,15-19).

Los tres primeros episodios poseen un sólido fundamento histórico por diversas razones. El primero, porque es bastante probable que Jesús conociera a algunos de sus futuros discípulos en el círculo del Bautista. El segundo, porque el dicho con que Jesús invita a Pedro y Andrés a que le sigan es con mucha probabilidad pre-pascual. Y el tercero, porque es bastante improbable que las comunidades cristianas inventaran una imagen tan negativa de Pedro ${ }^{7}$. La historicidad del

${ }^{6}$ Este pasaje no tiene paralelo en Lucas, pero sí en Mateo (Mt 16,13-23). Se trata de un paralelo muy elaborado, que claramente depende de Marcos en lo que se refiere a esta segunda llamada de Jesús a Pedro; véase: R. Aguirre, "La figura de Pedro en el evangelio de Mateo", en: Aguirre (ed.), Pedro en la iglesia primitiva... 43-59. pp. 47-51. También puede considerarse como paralelo el relato de Jn 6,66-71; véase: A. H. Maynard, "The Role of Peter in the Gospel of John" New Testament Studies 30 (1984) 531-548, pp. 533-534.

7 Sobre el primer pasaje véase: J. P. Meier, Un judio marginal. Nueva visión del Jesús histórico. Tomo II, 1: Juan y Jesús. El Reino de Dios (Estella: Verbo Divino 1999) pp. 163164. Sobre el segundo: J. Gnilka, El Evangelio según Marcos. Vol I: Mc 1,I-8,26 (Sala-

El discipulado contado en «iTú, sigueme!» 
cuarto episodio es mucho más discutible, pues parece reflejar una situación pospascual, en la que interesa mucho determinar las relaciones entre el Discípulo Amado y Pedro con respecto a Jesús ${ }^{8}$. Es importante tener en cuenta que estas cuatro tradiciones no sólo transmiten recuerdos históricos, sino también vivencias vocacionales de otros discípulos de las dos primeras generaciones cristianas que, al recordarlos y transmitirlos, los enriquecieron con sus propias experiencias ${ }^{9}$.

Los cuatro relatos pueden verse como diversos momentos de un mismo proceso espiritualvocacional
En cualquier caso, lo más interesante de estos testimonios vocacionales es que pueden ambientarse en diversos momentos de la relación de Pedro con Jesús. En cada caso, la experiencia vocacional abre una nueva etapa en el seguimiento, y en ese sentido todos ellos pueden leerse como diversos momentos de un mismo proceso.

Aun a riesgo de resultar un poco anacrónico, porque la percepción de la evolución personal en tiempos de Jesús era muy distinta de la que tenemos ahora, creo que es posible establecer una relación entre estos cuatro momentos del proceso vocacional de Pedro, y las principales fases del proceso espiritual, que suelen darse en distintas etapas de la vida. J. Garrido distingue cinco fases en la vida espiritual, que pueden servirnos de marco para situar proceso vocacional de Pedro. La primera corresponde al periodo de la adolescencia, y desde el punto de vista vocacional se caracteriza por la importancia de las mediaciones. La segunda corresponde a la fase vital del joven adulto, y vocacionalmente se caracteriza por una entrega libre y generosa a la voluntad de Dios. La tercera es una fase breve que corresponde a la crisis del realismo, y desde el punto de vista vocacional se caracteriza por la revisión de la etapa anterior y la búsqueda de nuevos horizontes. La cuarta corresponde a la etapa de la madurez, en la que se dejan atrás los

manca: Ed. Sígueme 1986) pp. 87-88. Y sobre el tercero: J. Gnilka, El evangelio según Marcos. Vol II: Mc 8,27-16,20 (Salamanca: Ed. Sígueme 1986) pp. 19-20.

8 Sobre la relación entre Pedro el Discípulo Amado en Jn 21 puede verse: Brown Donfried - Reuman, Pedro en el Nuevo Testamento... pp. 131-138; y: Maynard, "The Role of Peter... pp. 540-542.

${ }^{9}$ Es difícil establecer en qué medida se combinan en estos relatos de vocación la experiencia histórica de Pedro y la visión que tuvieron de él las primeras comunidades. En todo caso, la presentación de Pedro en Mc y en Jn 21 posee los rasgos de una experiencia individual. Véase T. Wiarda, "Peter as Peter in the Gospel of Mark" New Testament Studies 45 (1999) 19-37, espec. pp. 28-30 y 36; y: T. Wiarda, "John 21.1-23: Narrative Unity and its Implications," Journal for the Study of the NT 46 (1992) 53-71, espec. pp. 67-68. 
sueños de autorrealización, y desde el punto de vista vocacional se caracteriza por la unificación, simplificación y abandono confiado. Y la quinta se refiere al momento en que se transciende el proceso antropológico y supone una anticipación de la pascua ${ }^{10}$.

La vocación de Pedro en Judea podría ambientarse en la primera de estas fases. La llamada en Galilea posee los rasgos de la segunda. La invitación de Jesús a Pedro en Cesarea de Filipo y las instrucciones que siguen sobre el discipulado tienen muchos de los elementos que caracterizan la tercera y cuarta fases de esta tipología. Finalmente, la llamada del Resucitado a Pedro puede ambientarse en la última etapa. Es evidente que Pedro no pudo recorrer este complejo proceso en el tiempo que duró su relación con Jesús. No es eso lo que quiero decir, sino que estos cuatro relatos vocacionales poseen algunos rasgos característicos de las diversas etapas del proceso vocacional, y en cuanto tal pueden ayudarnos a entender nuestra propia experiencia vocacional como un proceso. Vamos a detenernos ahora en cada uno de ellos, teniendo como trasfondo el proceso del que forman parte.

\section{El primer encuentro (Jn 1,41-42)}

Este breve relato forma parte de una sección en la que se describe de forma paradigmática el proceso de fe de los discípulos (Jn 1,192,12). Dicho proceso comienza con el testimonio de Juan Bautista sobre sí mismo y sobre Jesús, y termina con una declaración del evangelista que señala el resultado final: "se manifestó su gloria y sus discípulos creyeron en él” (Jn 2,11). Este proceso transcurre a lo largo de siete jornadas (Jn 1,29.35.43; 2,1), dos de las cuales están dedicadas a narrar el encuentro de Jesús con sus cinco primeros discípulos. En la primera de ellas (Jn 1,35-42) se cuenta cómo Andrés y otro discípulo

En el primer encuentro

Andrés comunica a Pedro que han encontrado al Mesías

10 Garrido, Adulto y Cristiano... pp. 77-82, aunque conviene tener en cuenta toda la primera parte, en la que describe diversas formas de determinar las edades de la vida.

11 Haré una traducción bastante literal de los cuatro pasajes que voy a estudiar con el objeto de que puedan apreciarse algunos matices del texto griego. 
Encuentra éste (Andrés) a su propio hermano Simón y le dice: Hemos encontrado al Mesías, que significa Cristo. Le llevó a Jesús. Al verle Jesús dijo: tú eres Simón, el hijo de Juan, tú te llamarás Cefas, que significa Pedro.

Lo más llamativo en este pasaje es que la iniciativa no parte de Jesús, como en la mayoría de los relatos de vocación ${ }^{12}$. Tampoco parte de Pedro, sino de Andrés, que es también el primero en confesar a Jesús como Mesías. Esta forma de presentar las cosas podría responder al interés de Juan por relativizar la importancia de Pedro, pues esta presentación negativa es característica de Jn 1-20, es decir, de todo el evangelio antes de que se incluyera el apéndice de Jn $21^{13}$.

Es muy notable también la insistencia en los nombres: Mesías = Cristo; Cefas = Pedro; y sobre todo el hecho de que Jesús imponga a Simón un nuevo nombre. Sabemos que Pedro era conocido como Cefas en las comunidades cristianas de la primera generación, porque Pablo le llama así siete veces en sus cartas (1Cor 1,12; 3,22; 9,5; 15,5; Gál $1,18,2,9.11 .14)$, pero este es el único caso en que Pedro recibe tal nombre en los evangelios. El cambio de nombre aparece en otros dos pasajes: Mc 3,16 y Mt 16,17. En Mateo antecede a una declaración acerca de la autoridad de Pedro en la Iglesia (Mt 16,18-19), pero teniendo en

Importan-

cia del

cambio de

nombre:

Cefas $=$

Pedro cuenta la presentación que Juan hace de Pedro en el resto de su evangelio, es poco probable que tenga este sentido aquí. Más bien habría que pensar que Juan ha conservado el recuerdo del momento en que Jesús impuso este sobrenombre a Pedro, un hecho que también conoce Mar$\cos (\mathrm{Mc} 3,16)^{14}$. El mismo Marcos nos informa de que Jesús impuso otro sobrenombre a Juan y Santiago (Mc 3,17), de donde podemos

12 Nótese que el esquema de esta jornada es muy parecido al de la siguiente, pues también en ella Felipe (que en este caso ha sido llamado por Jesús), se encuentra a Natanael y le lleva hasta Jesús después de darle su testimonio sobre él (Jn 1,43-51); Véase: R. E. Brown, El evangelio según Juan (Madrid: Ed. Cristiandad 1979) pp. 269-270.

${ }^{13}$ Según Maynard, "The Role of Peter... pp. 543-546, esta presentación negativa responde a la polémica acerca del origen de la autoridad apostólica que se planteó en algunas iglesias cristianas a finales del siglo I. El grupo ecuménico que realizó un estudio conjunto sobre la figura de Pedro opina que la visión de Juan sobre Pedro no es tan negativa; véase: Brown - Donfried - Reuman, Pedro en el Nuevo Testamento... pp. 124-131.

14 Sobre el significado del nombre y las diversas interpretaciones que se han dado de este hecho puede verse: Aguirre, "La figura de Pedro... pp. 49-50, espec. la nota no 10. 
deducir que poner un sobrenombre a sus discípulos más allegados en el momento de la llamada podría haber sido una costumbre de Jesús.

La imposición de un nuevo nombre es uno de los rasgos que aparecen en los relatos de vocación, y está relacionado con una promesa o con un encargo (véase p.e. Gén 17,5). Sin embargo, en el caso de los discípulos de Jesús parece más bien que estos sobrenombres se refieren a algún rasgo de su carácter (firmeza de Pedro, impetuosidad de los Zebedeos), de modo que el hecho de imponerlos podría revelar el profundo conocimiento que Jesús tenía de ellos. Este podría ser el sentido que tiene en Juan, pues el hecho de conocer de antemano al discípulo aparece también resaltado en el pasaje paralelo del encuentro de Jesús con Nicodemo (Jn 1,47-50), y es uno de los rasgos de la relación maestro-discípulo en este evangelio, tal como aparece en la alegoría del buen pastor ${ }^{15}$.

Así pues, este episodio del evangelio de Juan recoge el recuerdo de un primer encuentro de Jesús con Pedro en el contexto del grupo de los seguidores del Bautista. Aunque Jesús no le llama como a Felipe, ni le invita a seguirle para ver dónde vive como a Andrés y al otro discípulo, su relación con él posee los rasgos propios de la relación maestro-discípulo, pues Jesús aparece como el buen pastor, que conoce a sus ovejas y las llama por su nombre (Jn 10,3.12). La actitud del Pedro en todo el relato es pasiva. De hecho es el único de los cinco discípulos mencionados en esta primera sección del evangelio de Juan que no responde a las palabras de Jesús con una confesión de fe o con el seguimiento.

Los rasgos que aparecen en este pasaje son característicos del comienzo de un proceso vocacional. Pedro inicia su relación con Jesús a través del testimonio de alguien cercano a él, alguien de quien puede fiarse. Andrés ha tenido una experiencia personal y honda de Jesús (esto es lo que significa en Juan "estar con"), se lo cuenta a su hermano y le lleva hasta Jesús. Su forma de actuar evoca la importancia de las mediaciones en este primer momento de la experiencia vocacional. También son propias de esta primera etapa la perplejidad de Pedro, que no responde ni reacciona, y la experiencia de saberse conocido en profundidad por Dios.

15 A. Destro - M. Pesce, "Il discepolato e l'iniziazione di Pietro nel vangelo de Giovanni”, en: L. Padovese (ed.) VI Simposio de Tarso su S. Paolo Apostolo (Roma: Istituto Francescano de Spiritualità - Pontificio Ateneo Antoniano 2000) 89-108, pp. 95-97.

Rasgos característicos del comienzo de un proceso vocacional

El nuevo
nombre relacionado con el conocimiento que tiene de ellos 
Este primer encuentro de Pedro con Jesús en clave vocacional inaugura, como los demás, una nueva etapa en su vida, pero no es todavía la etapa del seguimiento cercano. Tendrá que pasar algún tiempo para que vuelvan a encontrarse de nuevo en un contexto y una situación distintas. Este nuevo encuentro es el que Marcos refiere al comienzo de su evangelio.

\section{La respuesta de la juventud (Mc 1,16-18)}

Según Marcos, el primer encuentro de Pedro con Jesús tuvo lugar en Galilea, y no en Judea, algún tiempo después de que Juan Bautista fuera arrestado. Después de presentar a Jesús como una persona honorable (anunciado por Juan, declarado Hijo de Dios, y guiado por el Espíritu), describe el comienzo de su actividad pública en Galilea (Mc $1,14-3,6)$. En este contexto narra la llamada de los cinco primeros discípulos (Mc 1,16-20; 2,14), a los que más tarde se añadirán otros siete hasta completar el grupo de los Doce (Mc 3,13-19). La llamada de Pedro y su hermano Andrés es la primera de todas, y también lo primero que hace Jesús después de anunciar la llegada inminente del Reinado de Dios:

Caminando junto al mar de Galilea vio a Simón y a Andrés, el hermano de Simón, que estaban echando la red en el mar, pues eran pescadores. Jesús les dijo: Venid detrás de mí y os haré pescadores de hombres. Y dejando enseguida las redes le siguieron.

Llamada

de Jesús, respuesta inmediata de Pedro y Andrés
Es un relato muy esquemático, en el que todo sucede deprisa. Se subrayan, por un lado, la iniciativa de Jesús y su autoridad; y por otro, la respuesta inmediata de Pedro y Andrés. Es posible que Marcos lo haya situado al comienzo de su evangelio, precisamente, para resaltar estas dos cosas, y mostrar con un ejemplo cuál es la actitud con que deben acoger los oyentes/lectores del evangelio la invitación dè Jesús.

Marcos retoma aquí, muy probablemente una tradición oral, cuya forma literaria parece estar influenciada por el relato de la vocación de Eliseo. En él se cuenta cómo Elías encuentra a Eliseo arando y arroja sobre él su manto en señal de invitación; Eliseo deja entonces la yunta y se va detrás de Elías (1 Re 19,19-21) ${ }^{16}$. La existencia de una forma

16 Este relato ejerció un gran influyó en la formulación de las experiencias voca- 
literaria fija facilitó la transmisión y conservación de este episodio, pero al mismo tiempo plantea algunas preguntas acerca de su historicidad: ¿no será, acaso, una creación de las comunidades cristianas que idealizaron la respuesta de los primeros discípulos a la llamada de Jesús? ¿Hasta qué punto podemos fiarnos de datos que están tan vinculados al molde literario de este relato?

Sin embargo, la sospecha que encierran estas preguntas se desvanece cuando descubrimos algunos indicios que revelan detrás de esta formulación literaria estereotipada una experiencia histórica vivida por Pedro y Andrés. El dato más relevante son las palabras con las que Jesús les llama: "Venid detrás de mí y os haré pescadores de hombres". Jesús utiliza aquí una imagen que nunca antes había sido utilizada en el Judaísmo para referirse a una misión. Tampoco fue utilizada después en la Iglesia, que prefirió referirse a la tarea de los misioneros las imágenes del pastor o del jornalero (1 Pe 5,1-4; Lc 10,2)17. La imagen de la pesca posee, además, un colorido local muy preciso, pues sabemos con certeza que los primeros discípulos de Jesús eran pescadores. Por otro lado, el hecho sorprendente de que Pedro y Andrés siguieran inmediatamente a Jesús podría explicarse mejor si suponemos que le habían conocido anteriormente en el grupo del Bautista, como presupone el evangelio de Juan.

Como ya he dicho, los elementos más llamativos del relato son la autoridad con que Jesús llama y la prontitud con que Pedro y Andrés le responden. Este es el eje central del episodio, pero es también importante la ambientación. Jesús los encuentra en medio de sus ocupaciones, y ellos comprenden que la invitación a seguirle implica una ruptura con su vida anterior. La invitación que Jesús les dirige se refiere a dos momentos; uno presente: "veníos (ahora) detrás de mí" y otro futuro: "y os haré (después) pescadores de hombres". Seguir a Jesús no es la única finalidad de la llamada. Es importante también la misión. La combinación de ambas cosas explica la respuesta de los discípulos:

cionales de los primeros discípulos de Jesús (véase p.e. Mc 2,14 y Le 9,61-62); véase: J. Marcus, Mark 1-8. A New Translation with Introduction and Commentary (New York: Doubleday 2000) pp. 183-185.

17 Sobre la tradición y redacción de este pasaje, y su contextualización en el mundo de Jesús y del movimiento cristiano, puede verse: Guijarro Oporto, Fidelidades en conflicto... pp. 167-195.

«Venid detrás de mì. Imagen del discipulado

La invitación a seguirle implica ruptura con la vida anterior 
lo dejan todo y le siguen, porque es Jesús quien les llama, y porque les invita a participar en algo que merece la pena. Para entender este respuesta es necesario aclarar a qué se refiere exactamente Jesús con la expresión "pescadores de hombres".

La imagen de la pesca no es muy común en el AT ni en la literatura judía antigua, pero hay un pasaje que puede ayudarnos a entender su significado. Se encuentra en Jer 16,14-18, en un contexto de juicio. Dios enviará pescadores para que pesquen a su pueblo disperso por las naciones, y les traigan para dar cuentas de su conducta delante de él18. Jesús utiliza la imagen en el mismo sentido en una parábola que sólo se encuentra en el Evangelio de Mateo (Mt 13,47-50). En ella se compara la llegada del Reinado de Dios con una pesca en la que al final se seleccionan los peces buenos, y los malos se echan fuera. En esta parábola la imagen de la pesca se relaciona con la intervención escatológica de Dios. Tal vez por eso Marcos ha situado la llamada a Pedro y Andrés inmediatamente después de anunciar la llegada inminente del Reinado de Dios. El sentido de la imagen y el lugar en que Marcos ha situado esta invitación hacen suponer que Jesús estaba proponiendo a Pedro y a Andrés que participaran en el gran acontecimiento escatológico de la irrupción del Reinado de Dios. Una invitación de este tipo explica la prontitud e incondicionalidad con que le respondieron.

La experiencia vocacional reflejada en este pasaje es distinta a la del primer encuentro. El elemento dominante no es aquí el testimonio de otros, sino la tarea a la que Pedro y Andrés son invitados. Es una expe-

La experiencia dominante aquí es la tarea riencia vocacional propia de la etapa de la juventud adulta, que se caracteriza por la entrega libre y generosa a una tarea. Por eso la respuesta es limpia e inmediata, sin condiciones ni intermediarios. Nace de una relación personal y directa con Jesús, del reconocimiento de su autoridad sobre la propia vida, y sobre todo del entusiasmo que engendra la misión. En la edad de la juventud esta misión es lo que aparece en primer plano, y el polo que cataliza las energías de quien es llamado.

Con la respuesta entusiasta de Pedro se abre una nueva etapa en su proceso vocacional. Es la etapa del seguimiento explícito, marcada por el entusiasmo y la entrega sin condiciones a la misión. Esta es la

18 Sobre el uso de la imagen de la pesca en el AT y en la literatura de Qumran, véase: Wuellner, W., The Meaning of Fishers of Men (Philadelphia 1967) pp. 88-107. 
imagen de los discípulos que aparece a lo largo de la primera parte del Evangelio de Marcos (Mc 1,14-8,26): siguen a Jesús, están con él en todo momento, y son enviados por él como mensajeros del Reino. Sin embargo, a lo largo de estos capítulos comienzan a aparecer ciertas dificultades, hasta el punto de que a veces no comprenden a Jesús. Con el paso del tiempo y con el peso de la experiencia el camino de Pedro llegará a un punto crítico en el episodio de Cesarea de Filipo, con el que se abre una nueva etapa en su proceso vocacional.

\section{La crisis del realismo (Mc 8,27-33)}

El pasaje que recoge esta nueva experiencia vocacional de Pedro constituye un punto de inflexión en el relato de Marcos. Después de narrar la actuación de Jesús, acompañado siempre por sus discípulos, el relato entra en una nueva fase, que comienza con el planteamiento de dos cuestiones básicas: ¿Quién es Jesús? y ¿En qué consiste ser discípulo suyo? Son dos preguntas relacionadas, porque la respuesta a la segunda depende, en cierto modo, de lo que se haya respondido a la primera. Este nuevo planteamiento tiene que ver con el cambio de actitud que se da en los discípulos a lo largo de los capítulos precedentes (Mc 6,6b-8,26), en los que comienza a aparecer de forma reiterada su incomprensión ${ }^{19}$. Ahora la cuestión se plantea abiertamente, y es Pedro quien interviene como portavoz del grupo.

Salió Jesús con sus discípulos hacia las aldeas de Cesarea de Filipo, y en el camino preguntó a sus discípulos, diciéndoles: ¿Quién dice la gente que soy yo? Ellos respondieron: unos que Juan el Bautista y otros que Elías; otros incluso que uno de los profetas. él les preguntó: ¿Y vosotros, quien decís que soy yo? Pedro respondió diciéndole: Tú eres el Cristo. Y él los increpó para que no hablaran a nadie sobre él.

Y comenzó a enseñarles: es necesario que el Hijo del hombre sufra mucho y sea rechazado por los ancianos, por los jefes de los

${ }^{19}$ Los diversos pasajes en que aparece esta incomprensión (Mc 6,52; 7,18; 8,4. 1421) y su relación con la confesión de Pedro han sido estudiados minuciosamente por F. J. Matera, "The Imcomprehension of the Disciples and Peter's Confession (Mark 6,14-8,30)" Biblica 70 (1989) 153-172, aunque a mi modo de ver no insiste suficientemente en que dicha confesión encierra una nueva incomprensión, que hará necesaria una larga instrucción de Jesús a sus discípulos. 
sacerdotes y por los escribas, y que lo maten, y que resucite al cabo de tres días. Les hablaba de ello abiertamente.

Pedro, entonces, lo tomó aparte y comenzó a increparlo. Pero él, volviéndose hacia sus discípulos increpó a Pedro y le dijo: Ponte detrás de mí, Satanás, porque tus pensamientos no son los de Dios, sino los de los hombres.

He dividido el pasaje en tres párrafos para distinguir los tres momentos: confesión - anuncio de la pasión - incomprensión; y también para identificar gráficamente tres unidades tradicionales, que muy probablemente tuvieron una existencia independiente antes de que Marcos las uniera aquí20. Aunque la nueva llamada a Pedro se encuentra al final, haré un breve comentario de las otras dos secuencias para ambientar la tercera.

El diálogo de Jesús con sus discípulos recoge algunas opiniones que ya se han expresado antes en el evangelio (Mc 6,14-16). No es esto lo que le interesa a Marcos, sino la pregunta que Jesús dirige a sus propios discípulos. Pedro responde en nombre de todos afirmando que él es el Cristo, es decir, el Mesías esperado por Israel. A primera vista su respuesta es irreprochable (véase Mc 1,1), y por eso sorprende la reacción de Jesús, que los increpa, prohibiéndoles que hablen acerca de él. El verbo "increpar" que aparece tres veces en este contexto, es utilizado otras veces por Jesús para dirigirse a los demonios (véase: 1,25; $4,39 ; 9,25)$, y por eso no es extraño que más adelante Jesús le llame a Pedro Satanás. El mandato de no hablar sobre él y el uso de este verbo equiparan en cierto modo a los discípulos con los demonios, a los cuales Jesús también manda callar (Mc 1,25.34).

La reacción de Jesús indica que los discípulos no pueden respon-

Aún no pueden los discípulos responder adecuadamente der todavía adecuadamente a la pregunta que les ha planteado. Se hace necesaria una instrucción. Marcos dice expresamente que Jesús se puso a enseñarles, pero tanto el tono como el contenido de su enseñanza son nuevos. Jesús no les habla ahora de un Reino que irrumpe con poder en el mundo, sino de su muerte a manos de las autoridades judías; y tampoco les habla en parábolas, sino abiertamente. En esta nueva enseñanza su muerte se presenta como algo que responde al designio de Dios (este es el sentido de la expresión "es necesario"). Es

20 Gnilka, El evangelio según Marcos. Vol II... pp. 10-14. 
evidente que el anuncio de la pasión, tal como lo leemos en Marcos, es una profecía ex eventu formulada por los primeros cristianos, pero no es improbable que Jesús previera su muerte y hablara de ella a sus discípulos $^{21}$. En todo caso, en el relato de Marcos, esta forma de entender a Jesús y su destino se opone abiertamente a la que presupone la confesión de Pedro.

El contraste entre la confesión de Pedro y lo que Jesús acaba de proponer a sus discípulos se hace evidente en la tercera secuencia de la escena. Pedro es incapaz de aceptar a un Mesías que tenga que padecer en la cruz. A pesar de la descripción tan escueta, se advierte que su reacción es violenta: tomó aparte a Jesús y se puso a increparle, como si lo que acababa de decir se debiera a una inspiración diabólica. Es entonces cuando Jesús, utilizando el mismo tono, se dirige a él para conminarle a que vuelva a su puesto de discípulo: "ponte detrás de mí". Al increpar a Jesús para que abandone el camino de la cruz, no sólo ha abandonado el puesto de discípulo (detrás de Jesús), sino que se ha convertido en su tentador (Satanás). Es interesante observar que inmediatamente después de estas palabras Jesús inicia una instrucción dirigida a toda la gente y a los discípulos sobre el sentido preciso del seguimiento: "si alguien quiere seguir detrás de mí..." (Mc 8,34ss). La concentración redundante de estos dos términos del seguimiento indica de forma clara que la invitación de Jesús a Pedro "ponte detrás de mí" tiene un claro sentido vocacional.

La ambientación de estas palabras de Jesús a Pedro en el contexto actual es, muy probablemente, obra de Marcos o de algún otro catequista cristiano anterior a él. A ellos hay que atribuir la elaborada catequesis sobre el seguimiento que encontramos en esta sección del evangelio (Mc 8,31-10,52)22. Sin embargo, es muy probable que las pala-

21 Lo ha mostrado de forma convincente: H. Schürmann, ¿Cómo entendió y vivió Jesús su muerte? Biblioteca de Estudios Bíblicos 42 (Salamanca: Sígueme 1982).

22 La estructura básica de esta catequesis es fácilmente identificable, pues por tres veces se repite el mismo esquema literario: anuncio de la pasión - incomprensión de los discípulos manifestada de diversas formas - instrucción de Jesús. Esta repetición indica que la dificultad de los discípulos para entender el camino de Jesús (y el suyo propio) hacia la cruz es invencible desde el punto de vista humano, y que sólo Dios puede hacerles entender el sinsentido de un Mesías muerto en cruz. La escena final de esta catequesis, protagonizada por el ciego Bartimeo (Mc 10,46-52), pone de manifiesto esta situación, pues la única 
bras de Jesús conserven lo esencial de un dicho pronunciado por él, pues es impensable que los primeros cristianos inventaran una imagen tan negativa de Pedro. En este dicho de Jesús aparecen tres datos importantes que tienen que ver con la experiencia vocacional de Pedro. El primero es que su forma de afrontar el seguimiento en este momento se apoya en criterios humanos, y no en las categorías de Dios. En segundo lugar se dice que esta forma de actuar constituyó para Jesús una seria tentación. Y en tercer lugar, que Jesús tuvo que hacerle una nueva invitación al seguimiento.

No poseemos datos suficientes para ambientar históricamente este dicho, pero tanto su contenido como su tono encajan muy bien en un momento del ministerio de Jesús: la crisis que se dio en el grupo de sus discípulos. Esta situación puede deducirse a partir de varios indicios. Uno de ellos es este pasaje de Marcos. Otro es la escena que narra Juan al final del discurso del pan de vida, cuando muchos de sus discípulos le abandonan y él se dirige a los Doce para preguntarles si también ellos quieren marcharse. También aquí es Pedro quien responde a la pregunta de Jesús con una abierta confesión de fe (Jn 6,67-72). Finalmente, algunas parábolas que hablan del crecimiento misterioso del Reinado de Dios, como la parábola del sembrador o la de la semilla que crece sola, podrían haber sido pronunciadas para responder a una situación de crisis en el grupo de los discípulos, que no veían llegar dicho Reinado23. Esta crisis en el grupo de los discípulos afectaría sin duda a Pedro, y podría haber sido el contexto histórico de un dicho como éste.

En todo caso, como se trata de una reconstrucción muy hipotética, será mejor que nos atengamos a la presentación de Marcos, que por

manera de recobrar la vista para poder seguir de nuevo a Jesús por el camino, es pedírselo a él: "¡Señor, que vuelva a ver!". La situación inicial de Bartimeo (ciego, mendigo y al borde del camino) es la viva imagen de unos discípulos incapaces de entender el camino de Jesús para seguirle. Pero al mismo tiempo su actitud hacia Jesús es el camino para salir de ella, y reanudar el camino del seguimiento. Sobre el sentido catequético de este último pasaje y su relación con la catequesis que precede, véase: K. Kertelge, Die Wunder Jesu im Markusevangelium (München: Kösel 1970) 179-184.

23 Sobre el trasfondo histórico de Jn 6,67-72, véase: C. K. Barret, The Gospel according to Saint John (London: SPCK 19782 ed.) pp. 301-302. Sobre las parábolas que tratan de sostener la confianza de los discípulos: J. Jeremias, Las parábolas de Jesús (Estella: Verbo Divino 1976) pp. 179-188. 
otro lado resulta bastante verosímil. Al principio los discípulos siguieron a Jesús con entusiasmo, pero con el paso del tiempo aquel entusiasmo empezó a decaer y fueron apareciendo diversas formas de entender el camino de Jesús. El reconocimiento de que Jesús era el Mesías, lo mismo que la petición de los Zebedeos de sentarse uno a la derecha y otro a la izquierda en el reino que iba a inaugurar ( $\mathrm{Mc}$ 10,3540), revelan una concepción demasiado humana del proyecto de Jesús y el discipulado. Esta forma de entender el seguimiento dio lugar a una crisis vocacional reflejada en la reprensión de Pedro a Jesús, la cual a su vez hizo necesaria una nueva invitación a seguirle.

Es muy probable que Marcos haya querido establecer una relación entre la primera llamada a Pedro y Andrés: "Veníos detrás de mí" (Mc 1,17), y esta segunda dirigida sólo a Pedro: "Ponte detrás de mí" (Mc $8,33)$. Y es también probable que haya querido establecer un contraste entre ellas, pues en la primera llamada la invitación tenía una finalidad: "yo os haré pescadores de hombres", mientras que en la segunda esa referencia a la misión ha desaparecido. Este es el rasgo más característico de la segunda llamada: su concentración en el seguimiento, que adquiere nuevas connotaciones. No se trata ya sólo de acompañar a Jesús compartiendo su estilo de vida y su misión, sino que es necesario dar un paso más para compartir con él su destino. El objetivo de esta segunda llamada no es un proyecto, sino el mismo Jesús.

En este episodio encontramos los rasgos propios de la experiencia vocacional en la etapa de la madurez. El comienzo de esta etapa se caracteriza por una crisis de realismo, que supone una revisión crítica del pasado y un planteamiento abierto del futuro. Si esta crisis se supera, se inicia una etapa nueva en la vida espiritual, en la que se dejan atrás los sueños de autorrealización, y se vive el seguimiento como una experiencia de unificación, simplificación y abandono confiado. El pasaje que acabamos de comentar refleja muy bien el momento de la crisis del realismo, y abre el camino para esta nueva etapa del seguimiento.

La crisis del realismo sobreviene, según los autores espirituales, cuando se ha hecho un camino en el seguimiento cercano, después que hayan surgido las preguntas difíciles de responder, la incomprensión, o el fracaso. A estas alturas se ha experimentado la dureza del seguimiento, y en el horizonte de la vida aparece el realismo como una ten-

Momento de crisis: diversas formas de entender el camino

El objetivo de la segunda llamada es el mismo Jesús

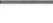


tación ${ }^{24}$. Esta tentación puede muy bien describirse con la frase de Jesús a Pedro: "tus pensamientos no son los de Dios, sino los de los hombres". Se difuminan en el horizonte las motivaciones de fe, y decae el entusiasmo por la misión.

Este momento es determinante en el proceso vocacional, porque

La identificación con Jesús determina el seguimiento maduro y adulto sólo entonces se dan las condiciones para que se produzca la "segunda llamada", y un seguimiento maduro y adulto. En esta segunda llamada lo decisivo no es la experiencia de otros, como en el primer encuentro; tampoco aparece en primer plano la tarea, como en la primera llamada. Lo central en este momento es la identificación con Jesús y el abandono a la voluntad de Dios. Esta es la llamada que invita a la segunda conversión en la mitad de la vida. Es en este momento cuando se percibe el alcance de las exigencias del seguimiento: renunciar a sí mismo, tomar la cruz, hacerse esclavo de los demás. Pero también es el momento en el que se experimenta una nueva unión con Jesús. La respuesta a esta segunda llamada consiste en quedarse sólo con él.

Con la respuesta a esta segunda llamada se inicia una etapa nueva en la experiencia vocacional de Pedro. El momento más representativo de esta nueva etapa en los evangelios lo encontramos en el relato de la pasión. Tanto Marcos como Juan presentan a Pedro como el discípulo que más decididamente siguió a Jesús durante su pasión, y también como aquel que le negó de forma más rotunda. Entre esos dos polos se sitúa su experiencia de seguimiento después de la crisis de Cesarea de Filipo, hasta que un nuevo encuentro con Jesús le revele cuál será el último tramo de su camino vocacional.

\section{Quedarse con lo esencial (Jn 21,15-19)}

La última experiencia vocacional de Pedro forma parte de un texto muy particular. Desde el punto de vista de la composición pare-

24 Uno de los autores espirituales que más se han ocupado de esta crisis en la vida espiritual es Johannes Tauler. Según él, suele darse en torno a los cuarenta años, y supone un giro en la vida. Es un momento de tentación (la huida y la inhibición son dos salidas frecuentes), pero sobre todo es una ocasión para iniciar una peregrinación hacia el "fondo del alma", y desde ahí entregar la propia voluntad a la voluntad de Dios. Una exposición clara y sugerente de la visión de Tauler sobre esta etapa de la vida espiritual puede verse en: A. Grün, La mitad de la vida como tarea espiritual. La crisis de los 40-50 años (Madrid: Narcea 1988) pp. 33-76. 
ce evidente que Jn 21 fue incorporado al evangelio de Juan algún tiempo después de su composición ${ }^{25}$. Sin embargo esto no implica necesariamente que todo el capítulo sea una composición tardía, ni que su intención original fuera establecer la primacía de Pedro sobre el Discípulo Amado ${ }^{26}$. Otros aspectos, entre ellos la notable unidad literaria del capítulo, inducen a pensar que antes de ser revisado para incluirlo como apéndice del evangelio de Juan, este breve relato contaba un encuentro de Jesús con Pedro, que tenía como tema central el discipulado $^{27}$. Este sigue siendo el tema central del capítulo, y uno de los efectos de su inserción en el evangelio de Juan consiste en haber creado una inclusión entre el comienzo y el final del mismo (Jn 1,35-50: 1lamada de los primeros discípulos; Jn 21,15-23: vocación de Pedro) ${ }^{28}$, de modo que todo el evangelio ha quedado enmarcado por estas dos experiencias vocacionales.

En este capítulo final hay dos invitaciones al seguimiento, pero la segunda es una reiteración de la primera, que es la que más nos interesa ahora. Se encuentra en la segunda escena del relato.

Cuando terminaron de comer le pregunta Jesús a Simón Pedro: Simón, hijo de Juan, ¿me amas más que estos?. El responde: Sí, Señor, tú sabes que te quiero. Le dice: Apacienta mis corderos. Le pregunta de nuevo por segunda vez: Simón, hijo de Juan, ¿me amas? Le responde: Sí, Señor, tú sabes que te quiero. Le dice: pastorea mi rebaño. Le pregunta por tercera vez: Simón, hijo de Juan, ¿me quieres? Se entristeció Pedro porque le había dicho la tercera vez ¿me quieres? Y le dijo: Señor, tú sabes que te quiero. Le dice Jesús: Apacienta mi rebaño. En

25 Es una convicción compartida por los estudiosos de Juan; véase p. e. Brown Donfried - Reuman, Pedro en el Nuevo Testamento... pp. 131-132.

${ }^{26}$ Esta es una opinión muy difundida, y de hecho está presente en la redacción final. Sin embargo, es interesante observar que dicha relación se plantea sólo al final del capítulo, lo cual indica que se trata de un tema secundario. Tal vez no era tan importante en el relato tradicional.

27 Wiarda, "John 21.1-23... pp. 68-69.

28 M. Franzmann - M. Klinger, "The Call Stories of John 1 and John 21" St. Vladimir Theological Quarterly 36 (1992) 7-15, han estudiado las relaciones entre Jn 1,35-50 y Jn 21,15-23, y han mostrado que la llamada inicial de Pedro queda incompleta sin esta última invitación. Por su parte, Destro - Pesce, "Il discepolato e l'iniziazione di Pietro... p. 104, señalan que sólo en Jn 1,42 y 21,17 se menciona a Juan con el nombre de "Simón, hijo de Juan", lo cual es un indicio más de la relación entre estos dos relatos vocacionales. 
Es evidente que los autores de los evangelios no pensaron en relacionar estos cuatro episodios con las diversas etapas de la vida, ni tampoco con las diversas fases de la vida espiritual. Y sin embargo, es también evidente que en ellos han quedado reflejados momentos muy diversos de la experiencia de Pedro como discípulo de Jesús. No debemos olvidar que estos recuerdos fueron conservados por los cristianos de las dos primeras generaciones cristianas, y que al conservarlos y transmitirlos los fueron enriqueciendo con su propia experiencia. Como tuvieron ocasión de leerlos en diversos momentos de su propio proceso vocacional, no es improbable que hayan dejado en ellos retazos de cómo vivieron los diversos momentos de dicho proceso.

Para concluir, resumiré de forma descriptiva el proceso descrito en estos cuatro relatos, resaltando lo característico de cada uno de ellos. El primer encuentro de Jesús con Pedro en Judea (Jn 1,41-42) evoca la experiencia vocacional de la adolescencia. Lo más importante en este primer momento es el testimonio de otros y el hecho de sentirse conocido en profundidad por Jesús, pero todavía no hay una llamada explícita. La primera llamada tiene lugar en el segundo encuentro (Mc 1,1618). Esta primera llamada está centrada en la tarea, y se caracteriza por una entrega total a la causa del Reinado de Dios. La respuesta a esta

Proceso:

- adoles-

cencia

- juventud

- madurez

- vejez llamada es la propia del tiempo de la juventud, cuando todavía no se hacen cálculos y todo parece posible junto a Jesús. Ahora bien, con el paso del tiempo las experiencias negativas se van acumulando, van surgiendo las preguntas y se impone el realismo. El discípulo se encamina así hacia una crisis muy profunda y llena de tentaciones. Es entonces cuando se escucha la segunda llamada (Mc 8,33), una llamada que no tiene ya su centro en la misión, sino en el mismo Jesús, y que tiende a la aceptación serena del proyecto de Dios sobre la propia vida. Se abre así una nueva etapa no exenta de contradicciones (negaciones), que aboca a una nueva llamada, ya al final de la vida (Jn 21,15-19), en la que el discípulo se va quedando con lo único perdurable: un amor como el de Jesús. 